\title{
Qualidade seminal e morfometria testicular de coelhos (Oryctolagus cuniculus) suplementados com geleia real
}

\author{
[Seminal quality and testicular morphometry of rabbits (Oryctolagus cuniculus) \\ supplemented with royal jelly] \\ P.K.A. Campos ${ }^{1}$, L.P. Barbosa ${ }^{2 *}$, M.M. Neves ${ }^{1}$, B.E.S. Melo ${ }^{1}$, A.C.T. Morais ${ }^{1}$, D.B. Morais ${ }^{1}$ \\ ${ }^{1}$ Universidade Federal de Viçosa - Viçosa, MG \\ ${ }^{2}$ Universidade Federal do Recôncavo da Bahia - Cruz das Almas, BA
}

\begin{abstract}
RESUMO
Objetivou-se avaliar o efeito da geleia real na qualidade seminal e na morfometria testicular de coelhos. Quatorze coelhos adultos da raça Nova Zelândia foram distribuídos em três grupos: com administração diária de $1 \mathrm{~mL}$ de água, via oral (SG); administração diária de $0,5 \mathrm{mg}(0,5 \mathrm{G})$ de geleia real diluída em $1 \mathrm{~mL}$ de água, via oral; e administração diária de $1,0 \mathrm{~mL}(1,0 \mathrm{G})$ de geleia real diluída em $1 \mathrm{~mL}$ de água, via oral. $\mathrm{O}$ fornecimento de geleia real foi iniciado 30 dias antes das coletas de sêmen, permanecendo durante todo o período de coleta, totalizando 90 dias. Utilizou-se o método da vagina artificial para coleta de sêmen. Foram avaliados os parâmetros físicos e morfológicos do sêmen e os parâmetros de morfometria testicular. Houve diferença no volume seminal do $0,5 \mathrm{G}(0,54 \pm 0,22)$ em relação ao $\mathrm{SG}(0,39 \pm 0,13)$ e ao 1,0G $(0,30 \pm 0,09)(\mathrm{P}<0,05)$. Para os grupos $\mathrm{SG}, 0,5 \mathrm{G}$ e $1,0 \mathrm{G}$, não houve diferença $(\mathrm{P}>0,05)$ para turbilhonamento espermático, concentração espermática, motilidade progressiva e vigor espermático. Os defeitos maiores no grupo $0,5 \mathrm{G}(8,52 \pm 3,26)$ foram menores do que nos grupos $\mathrm{SG}(14,09 \pm 4,26)$ e $1,0 \mathrm{G}$ $(16,1 \pm 3,95)(\mathrm{P}<0,05)$. Não houve diferença entre os defeitos menores e os defeitos totais $(\mathrm{P}>0,05)$. Os pesos corporal, testicular, epididimário e o índice gonadossomático não diferiram entre os grupos $(\mathrm{P}>0,05)$. A ingestão diária de $0,5 \mathrm{mg}$ de geleia real apresentou efeitos positivos na morfologia espermática de coelhos.
\end{abstract}

Palavras-chave: índice gonadossomático, patologia espermática, sêmen

\begin{abstract}
A trial was carried out to evaluate the effects of royal jelly on the seminal quality and testicular morphometry of rabbits. Fourteen mature rabbits of New Zealand breed were distributed between three groups. The first group was supplied with $1 \mathrm{~mL}$ of water only $(S G)$, the second group was supplied with $0.5 \mathrm{mg}$ of royal jelly diluted in $1 \mathrm{~mL}$ of water $(0.5 \mathrm{G})$, and the third group was supplied with $1 \mathrm{mg}$ of royal jelly also diluted in $1 \mathrm{~mL}$ of water (1.0G). The royal jelly supply started 30 days before semen collection and lasted the entire experimental period. An artificial vagina was used to collect the rabbits' semen. Physical and morphological parameters in the semen and the testicular morphometry were evaluated. Differences were found on the seminal volume in group $0.5 G(0,54 \pm 0,22)$ in relation to $S G(0,39 \pm 0,13)$ and $1.0 G(0,30 \pm 0,09)$ groups $(P<0.05)$. For $S G, 0.5 G$ and $1.0 G$ groups, no differences $(P>0.05)$ were found in sperm concentration, gross motility, individual motility and vigor. The total of primary defects in group $0.5 G(8,52 \pm 3,26)$ was lower than in groups $S G(14,09 \pm 4,2)$ and $1.0 G(16,1 \pm 3,95)(P<0.05)$. No significant difference was found between secondary defects and the total defects on the semen $(P>0.05)$. Body, testicular and epididymal weights did not differ between groups, as well to the gonadosomatic index $(P>0.05)$. The ingestion of royal jelly produced positive results on the seminal production of males.
\end{abstract}

Keywords: gonadosomatic index, semen, spermatic pathology

Recebido em 5 de abril de 2011

Aceito em 3 de abril de 2012

*Autor para correspondência (corresponding author)

E-mail: larissa@ufrb.edu.com.br 


\section{INTRODUÇÃO}

Nos últimos anos, é perceptível um interesse maior em todo o mundo pelos produtos apícolas, incluindo a geleia real, justificado pelo fato de estes produtos apresentarem propriedades medicinais e nutritivas singulares. Várias análises químicas e testes para as atividades biológicas da geleia real e sua composição têm sido relatados na literatura (Rocha et al., 2003).

A geleia real possui em sua composição $63 \%$ de umidade, 3,5\% de lipídios (esteroides), $13 \%$ de proteínas e $19,5 \%$ de carboidratos, minerais, e vitaminas (tiamina, riboflavina e niacina) (Amoedo e Muradian, 2002) e outros componentes ainda não identificados (Kohno et al., 2004). O ácido 10-hidroxi-2-decenoico (10HDA) é o principal componente da sua fração lipídica e é considerado o mais importante princípio ativo da geleia real (Koshio e Muradian, 2003).

Segundo Koshio e Muradian (2003), a fertilidade e o longo período de vida da abelha rainha, que é alimentada exclusivamente com geleia real, tem gerado interesse pelo consumo desse produto, por causa de seus prováveis efeitos medicinais para problemas hepáticos, cardíacos, imunológicos, dermatológicos e para questões relacionadas à reprodução, como aumento da fertilidade e libido.

Alguns estudos retratam o uso da geleia real atuando no processo reprodutivo de animais (Khattab et al., 1988; Husein e Haddad, 2006; Morais et al., 2009; Barbosa et al., 2009). Em coelhos, o tratamento com geleia demonstrou uma melhora da fertilidade e do desenvolvimento embriogênico (Khattab et al., 1988). Husein e Haddad (2006), em seus experimentos, concluíram que a geleia real aumenta o desempenho reprodutivo em ovelhas. Embora os mecanismos de ação desta substância ainda não estejam determinados, ela possui efeitos positivos no desenvolvimento e crescimento folicular e na gestação, sustentando a hipótese de que a geleia real produza efeitos similares aos produzidos pela gonadotrofina coriônica equina. Kridli e Al-Khetib (2006) concluíram que a geleia real pode ter efeitos positivos na gestação e na taxa de nascidos, agindo de maneira a melhorar a resposta ao estro e a taxa de concepção, com o aumento do desenvolvimento folicular, resultando na maior produção de estrógeno.

Considerando-se que são escassos estudos para testar a hipótese de que a geleia real age de maneira positiva nos aspectos reprodutivos de machos, objetivou-se avaliar o efeito da geleia real in natura nos parâmetros físicos e morfológicos do sêmen e na morfometria testicular de coelhos Nova Zelândia (Oryctolagus cuniculus).

\section{MATERIAL E MÉTODOS}

O projeto foi realizado no Biotério do Centro Universitário do Leste de Minas Gerais Unileste-MG, em Coronel Fabriciano-MG. Foram utilizados 14 coelhos machos adultos (Oryctolagus cuniculus) da raça Nova Zelândia. Os animais foram mantidos sob as mesmas condições ambientais, com temperatura a $24^{\circ} \mathrm{C}$ e luminosidade controlada, sendo o fotoperíodo de 12 horas de luz e 12 horas de escuridão. Receberam ração balanceada e água ad libitum. Foram alojados em gaiolas de metal, própria para coelhos, com lotação de dois animais por gaiola.

Os animais foram distribuídos aleatoriamente em três grupos, sendo: administração de $1 \mathrm{~mL}$ de água por via oral, diariamente $(\mathrm{n}=4)(\mathrm{SG}$, grupocontrole); administração de $0,5 \mathrm{mg}$ de geleia real diluída em $1 \mathrm{~mL}$ de água via oral, diariamente $(\mathrm{n}=5)(0,5 \mathrm{G})$; e administração de $1 \mathrm{mg}$ de geleia real diluída em $1 \mathrm{~mL}$ de água via oral, diariamente $(\mathrm{n}=5)(1,0 \mathrm{G})$.

A suplementação dos animais com geleia real foi iniciada 30 dias antes das coletas de sêmen, permanecendo até o final do período experimental, totalizando três meses de suplementação.

O sêmen foi coletado duas vezes por semana durante dois meses, utilizando-se o método de vagina artificial, com outro macho como manequim. Foi usada uma caixa de coleta, apresentando $80 \mathrm{~cm}$ de comprimento, $40 \mathrm{~cm}$ de largura e $30 \mathrm{~cm}$ de altura. Após a coleta do sêmen, este foi imediatamente levado ao banhomaria, estabilizado à temperatura de $37^{\circ} \mathrm{C}$, seguindo-se à realização do seu exame físico.

Foram analisados os parâmetros físicos do sêmen como: aspecto seminal; volume seminal; 
concentração espermática, avaliada em câmara de Neubauer; turbilhonamento espermático (0-5); motilidade espermática progressiva (0-100\%); vigor espermático (0-5), bem como os parâmetros morfológicos do sêmen, por meio de avaliações das patologias espermáticas, classificadas como defeitos maiores e defeitos menores e defeitos totais, segundo o Colégio Brasileiro de Reprodução Animal (CBRA) (Fonseca et al., 1998).

Após o período de suplementação e o de avaliação seminal, os coelhos foram eutanasiados, utilizando-se aplicação intravenosa de tiopental, diluído em soro fisiológico e éter. Posteriormente os animais foram pesados, e os testículos retirados. Estes órgãos, direito e esquerdo, bem como o epidídimo, foram dessecados e pesados em balança de precisão. Por meio do peso de ambos os testículos, foi possível calcular o índice gonadossomático, o qual se refere ao percentual de massa corporal alocado em gônadas (Costa et al., 2004).

Usou-se um delineamento inteiramente ao acaso, e, para análise dos dados, foi utilizado o teste de Kruskal-Wallis para avaliação de turbilhonamento espermático, vigor espermático, volume seminal, motilidade espermática e peso testicular, bem como a análise de variância (ANOVA) para concentração espermática, patologias espermáticas (defeitos primários e defeitos secundários), peso corporal, peso epididimário e índice gonadossomático, utilizando-se $5 \%$ de probabilidade.

\section{RESULTADOS E DISCUSSÃO}

Os ejaculados analisados, tanto no grupocontrole quanto nos grupos tratados com geleia real, apresentaram coloração leitosa, o que é característico da espécie. Das amostras analisadas, apenas uma do grupocontrole apresentou coloração amarelo-citrina, característica de ejaculado pouco concentrado.

Houve diferença para o volume seminal entre os grupos (Tab. 1), sendo que os animais do 0,5G apresentaram valores superiores $(0,54 \pm 0,22) \mathrm{em}$ relação aos animais do $1,0 \mathrm{G}(0,30 \pm 0,09)$ e aos animais do $\mathrm{SG}(0,39 \pm 0,13)(\mathrm{P}<0,05)$.

Tabela 1. Avaliação física seminal de coelhos suplementados com geleia real in natura

\begin{tabular}{lccc}
\multicolumn{1}{c}{ Parâmetros } & SG & $0,5 \mathrm{G}$ & $1,0 \mathrm{G}$ \\
\hline Volume seminal (mL) & $0,39 \pm 0,13 \mathrm{~b}$ & $0,54 \pm 0,22 \mathrm{a}$ & $0,30 \pm 0,09 \mathrm{~b}$ \\
Turbilhonamento espermático (0 a 5) & $3,00 \pm 1,58$ & $3,33 \pm 1,12$ & $3,11 \pm 1,17$ \\
Concentração espermática (x10\% $/ \mathrm{mL})$ & $147,44 \pm 01,27$ & $132,94 \pm 20,88$ & $100,99 \pm 09,24$ \\
Motilidade espermática (\%) & $62,22 \pm 38,98$ & $78,33 \pm 23,18$ & $73,89 \pm 24,59$ \\
Vigor espermático (0 a 5) & $3,00 \pm 1,73$ & $3,67 \pm 1,12$ & $3,56 \pm 1,24$ \\
\hline
\end{tabular}

$\mathrm{SG}=$ sem geleia real; $0,5 \mathrm{G}=0,5 \mathrm{mg}$ de geleia real; $1,0 \mathrm{G}=1 \mathrm{mg}$ de geleia real.

Valores na linha seguidos por letras diferentes diferem entre si pelo teste de Kruskal-Wallis para turbilhonamento espermático, volume seminal, motilidade progressiva e vigor espermático, e pela ANOVA para concentração espermática, a 5\% de probabilidade.

As médias dos volumes seminais encontradas neste experimento são similares aos valores encontrados por Oliveira et al. (2004), em seu estudo com a suplementação de zinco em coelhos. Andreazzi et al. (2004) obtiveram média de volume de $0,59 \mathrm{~mL}$ em seu experimento com coelhos Nova Zelândia, avaliando a qualidade do sêmen dos animais alimentados com rações contendo diferentes fontes de óleos vegetais.

O ejaculado do coelho tem volume amplamente variado, desde 0,3 a 6mL (Badú, 2003; Oliveira, 2004). Neste estudo, a média do volume seminal encontra-se dentro da faixa considerada normal para a espécie, indicando o bom funcionamento das glândulas sexuais acessórias. Em seu estudo com coelhos, Foote (1999) ressalta que o volume do ejaculado é evidência do normal funcionamento das glândulas acessórias, associado à produção de andrógenos e à boa libido do animal. Desta forma, a produção de andrógenos pode ter sido favorecida no grupo $0,5 \mathrm{G}$, sendo refletida no aumento de volume seminal.

Os valores de turbilhonamento espermático não apresentaram diferenças significativas $(\mathrm{P}>0,05)$ entre o grupo-controle $(3 \pm 1,58)$ e os grupos tratados com geleia real, $0,5 \mathrm{G}(3,33 \pm 1,12)$ e 1,0G $(3,11 \pm 1,17)$ (Tab. 1). 
Holtz e Foote (1978), em seus estudos analisando a constituição e diversos parâmetros do sêmen de coelhos Nova Zelândia, obtiveram 3,3 $\pm 1,2$ de turbilhonamento espermático. Shimamoto e Sofikitis (1998), para este mesmo parâmetro, encontraram $2,3 \pm 0,4$ no grupo tratado com dieta rica em colesterol e valor de $3,3 \pm 0,3$ para o grupo- controle, sendo este valor similar aos obtidos neste experimento.

As médias de concentração espermática não diferiram significativamente $(\mathrm{P}>0,05)$ entre os tratamentos (Tab. 1). Observa-se que houve uma variação nos valores de desvio- padrão. Holtz e Foote (1978) obtiveram uma média de concentração espermática de $90,2 \times 10^{6} / \mathrm{mL}$, similar aos valores encontrados neste estudo. Entretanto, Andreazzi et al. (2004) encontraram valores maiores, de $207 \times 10^{6} / \mathrm{mL}$.

Não houve diferença na motilidade espermática progressiva e no vigor espermático do sêmen dos animais nos três grupos $(\mathrm{P}>0,05)$ (Tab. 1), mostrando que a geleia real não influenciou nos referidos parâmetros.

Badú (2003) encontrou um valor médio de $85,6 \%$ de motilidade espermática, obtendo valor maior do que o encontrado neste estudo. Para vigor espermático, obteve um valor de 3,4 em seu experimento, sendo que as coletas foram realizadas na mesma estação do ano do presente experimento.

Os resultados obtidos por Oliveira (2004) em coelhos, utilizando suplementação com zinco, não diferiram dos achados neste estudo, pois foram encontrados valores de motilidade espermática progressiva de 73,60 a 79,17\% e vigor espermático de 3,4 a 4,0. Shimamoto e Sofikitis (1998) obtiveram $79 \%$ de motilidade espermática.

A média de motilidade espermática obtida por Holtz e Foote (1978) foi relativamente baixa, comparando-a com este experimento, com um valor de 58\%, semelhante aos resultados apresentados por Andreazzi et al. (2004), em que a média da motilidade espermática progressiva foi de $49 \%$, considerada baixa.

Não houve diferença entre os defeitos secundários e os defeitos totais nos três grupos avaliados $(\mathrm{P}>0,05)$ (Tab. 2). Entretanto, o total de defeitos primários no $0,5 \mathrm{G}(8,52 \pm 3,26)$ foi significativamente menor do que no SG $(14,09 \pm 4,26)$ e no $1,0 \mathrm{G}(16,10 \pm 3,95)$. Essa diferença pode ser um reflexo também do efeito da geleia real na produção de andrógenos, influenciando diretamente na eficiência do processo de espermatogênese.

Tabela 2. Avaliação morfológica do sêmen de coelhos suplementados com geleia real in natura

\begin{tabular}{|c|c|c|c|}
\hline Parâmetro & $\mathrm{SG}$ & $0,5 \mathrm{G}$ & $1,0 \mathrm{G}$ \\
\hline Defeitos primários & $14,09 \pm 4,26 b$ & $8,52 \pm 3,26 a$ & $16,10 \pm 3,95 b$ \\
\hline Defeitos secundários & $22,88 \pm 5,26$ & $21,78 \pm 3,64$ & $19,78 \pm 7,08$ \\
\hline Defeitos totais & $36,97 \pm 6,58$ & $30,33 \pm 2,12$ & $35,88 \pm 7,04$ \\
\hline
\end{tabular}

$\mathrm{SG}=$ sem geleia real; $0,5 \mathrm{G}=0,5 \mathrm{mg}$ de geleia real; $1,0 \mathrm{G}=1 \mathrm{mg}$ de geleia real.

Valores na mesma linha seguidos por letras diferentes diferem entre si pela análise de variância, a $5 \%$ de probabilidade.

Ressalta-se que os tipos de patologias de defeitos primários mais visualizadas nos três grupos foram gota citoplasmática proximal e contorno anormal da cabeça. Os valores encontrados são similares aos obtidos por Holtz e Foote (1978), que, em estudo com sêmen de coelhos Nova Zelândia, obtiveram uma proporção de espermatozoides com gota citoplasmática proximal sempre menor que $10 \%$ e afirmam que a aglutinação de cabeças de espermatozoides é comum nas amostras. Entre as patologias observadas em seu estudo, Badú (2003) discriminou as observadas na membrana acrossômica devido a sua importância na fertilização, em virtude de sua alta ocorrência.

Os valores de peso corporal, peso testicular, peso epididimário e de índice gonadossomático não apresentaram diferença entre os grupos avaliados $(\mathrm{P}>0,05)$ (Tab. 4). 
Qualidade seminal...

Tabela 4. Peso corporal, peso testicular, peso epididimário e de índice gonadossomático de coelhos suplementados com geleia real in natura

\begin{tabular}{lccc}
\multicolumn{1}{c}{ Parâmetros } & $\mathrm{SG}$ & $0,5 \mathrm{G}$ & $1,0 \mathrm{G}$ \\
\hline Peso corporal $(\mathrm{Kg})$ & $3,20 \pm 0,18$ & $2,95 \pm 0,29$ & $3,07 \pm 0,33$ \\
Peso testículo direito $(\mathrm{g})$ & $2,35 \pm 0,33^{*}$ & $2,53 \pm 0,33^{*}$ & $2,03 \pm 0,93^{*}$ \\
Peso testículo esquerdo $(\mathrm{g})$ & $2,38 \pm 0,49^{*}$ & $2,54 \pm 0,32^{*}$ & $1,89 \pm 1,01^{*}$ \\
Peso epidídimo direito $(\mathrm{g})$ & $1,12 \pm 0,24$ & $1,28 \pm 0,30$ & $1,01 \pm 0,19$ \\
Peso epidídimo esquerdo $(\mathrm{g})$ & $1,02 \pm 0,25$ & $1,26 \pm 0,27$ & $0,97 \pm 0,33$ \\
Índice gonadossomático $(\%)$ & $0,14 \pm 0,020$ & $0,17 \pm 0,027$ & $0,12 \pm 0,058$ \\
\hline
\end{tabular}

$\mathrm{SG}=$ sem geleia real; $0,5 \mathrm{G}=0,5 \mathrm{mg}$ de geleia real; $1,0 \mathrm{G}=1 \mathrm{mg}$ de geleia real.

Os parâmetros avaliados não diferiram entre si pela análise de variância e pelo teste de Kruskal-Wallis*, a 5\% de probabilidade.

Os pesos dos animais assemelham-se ao descrito para esta espécie e aos valores obtidos por Foote (1999). Castro et al. (2002) obtiveram peso corporal dos coelhos de seus estudos um pouco maior, em torno de 4,29kg. Shimamoto e Sofikitis (1998), em seus estudos avaliando o alto nível de colesterol na função testicular e no sêmen de coelhos, encontraram peso testicular em $\mathrm{kg}$ de $3,153 \pm 1,01$ no grupo tratado com alta concentração de colesterol e de 3,208 $\pm 0,94$ no grupo-controle.

O índice gonadossomático no SG foi de $0,14 \%$, e de $0,17 \%$ e $0,12 \%$, no $0,5 \mathrm{G}$ e no $1,0 \mathrm{G}$, respectivamente. Este índice representa o percentual de massa corporal alocado em testículos, que foi expresso em percentual.

Segundo Costa et al. (2004), várias teorias buscam explicar por que uma espécie aloca proporção de massa corporal em testículos mais que outras. A mais aceita baseia-se no comportamento reprodutivo, ou seja, na frequência de cópulas dos reprodutores.

\section{CONCLUSÕES}

A ingestão diária de $0,5 \mathrm{mg}$ de geleia real aumentou o volume seminal e diminuiu os valores de defeitos espermáticos primários de coelhos Nova Zelândia, apresentando efeitos positivos na morfologia seminal.

\section{AGRADECIMENTOS}

À Fundação de Amparo à Pesquisa do Estado de Minas Gerais (FAPEMIG), pelo financiamento da pesquisa.

\section{REFERÊNCIAS}

AMOEDO, L.H.G.; MURADIAN, L.B.A. Comparação de métodos para a determinação de umidade em geleia real. Química Nova, v.25, p.676-679, 2002.

ANDREAZZI, M.A.; SCAPINELLO, C.; MORAES, G.V. et al. Avaliação da qualidade do sêmen em coelhos alimentados com rações contendo diferentes fontes de óleos vegetais. Acta Sci. Anim. Sci., v.26, p.87-93, 2004.

BADÚ, C.A. Eficiência reprodutiva de coelhas inseminadas artificialmente e mantidas em sistemas semi-intensivo e extensivo de produção. 2003. 38f. Dissertação (Mestrado em Zootecnia). UFMG. 2003.

BARBOSA, L.P.; RODRIGUES, M.V.; BALARINI, M.K. et al. Qualidade embrionária de camundongos (Mus musculus) suplementados com geleia real. Rev. Bras. Saúde Prod. Anim., v.10, p.146-152, 2009.

CASTRO, A.C.S.; BERNDTSON, W.E.; CARDOSO, F.M. Plasma and testicular testosterone levels, volume density and number of Leydig cells and spermatogenic efficiency of rabbits. Braz. J. Med. Bio. Res. v.35, p.493-498, 2002.

COSTA, D.S.; HENRY, M.R.G.M.; PAULA, T.A.R. Espermatogênese de catetos (Tayassu tajacu). Arq. Bras. Med. Vet. Zoot., v.56, p.4951, 2004.

FONSECA, C.A.; FILHO, V.R.M.; FILHO, A.M. et al. Procedimentos para exame andrológico e avaliação de sêmen animal. Colégio Brasileiro de Reprodução AnimalCBRA, Belo Horizonte.1992. 82p. 
FOOTE, R.H. Cadmium affects testes and semen of rabbits exposed before and after puberty. Reprod. Tox., v.13, p.269-277, 1999.

HOLTZ, W.; FOOTE, R.H. Composition of rabbit semen and the origin of several constituents. Bio. Reprod., v.18, p.286-292, 1978.

HUSEIN, M.Q.; HADDAD, S.G. A new approach to enhance reproductive performance in sheep using royal jelly in comparison with equine chorionic. Anim. Reprod. Sci., v.93, p.2433, 2006.

KHATTAB, M.M.; RADWAN, A.A.; AFIFI, E.A. Physiological effect of Royal jelly on female reproductive capacity in rabbits. In: INTERNATIONAL CONGRESSO IN APICULTURE IN TROPICAL CLIMATE, 4. 1988, Cairo. Proceedings...Cairo: IBRA, London, UK. p.6-10, 1988.

KOSHIO, S.; MURADIAN, L.B. Aplicação da clae para determinação do ácido 10-hidróxi-2decenoico (10-HDA) em geleia real pura e adicionada a mel brasileiro. Química Nova. v.26, p.670-673, 2003.

KOHNO, K.; OKAMOTO, I.; SANO, O. et al. Royal jelly inhibits the production of proinflammatory cytokines by activated macrophages. Biosci. Biotechnol. Biochem., v.68, p.138-145, 2004.
KRIDLI, R.T.; AL-KHETIB, S.S. Reproductive responses in ewes treated with eCG or increasing doses of royal jelly. Anim. Reprod. Sci., v.92, p.75-85, 2006.

MORAIS, A.C.T.; BARBOSA, L.P.; NEVES, M.M. et al. Parâmetros morfofisiológicos testiculares de camundongos (Mus musculus) suplementados com geleia real. Arq. Bras. Med. Vet. Zootec., v.61, p.110-118, 2009.

OLIVEIRA, C.E.A. Avaliação seminal $e$ utilização digestiva em coelho reprodutores submetidos a dietas com diferentes níveis de zinco. 2004. 36f. Dissertação (Mestrado em Zootecnia). UFMF - Departamento de Veterinária. 2004.

ROCHA, H.C.; RAMOS, P.R.R.; FUNARI, S.R.C. Eletroferograma de proteínas de glândulas hipofaringeanas de abelhas Apis mellifera L. submetidas à produção de geleia real. Boletim da Indústria Animal, v.60, p.39-46, 2003.

SHIMAMOTO, K.; SOFIKITIS, N. Effect of hypercholesterolaemia on testicular function and sperm physiology. Yonago Acta Med., v.41, p.23-29, 1998. 